\title{
El otro como objeto: biopolítica, transpolítica y diagnóstico
}

\section{The other as an object: biopolitic, transpolic and diagnostic}

\author{
América Celeste Guevara Parra \\ Psicóloga por la Universidad Autónoma de Bucaramanga; magister (c) en Antropología Social por la \\ Universidad Autónoma de Barcelona, España \\ Correo electrónico: aguevara2@unab.edu.co
}

\begin{abstract}
Resumen
En Occidente a finales del siglo XVIII se experimenta la paulatina inserción del poder político en la vida biológica de la población, se trata de un control que se ejerce con el objetivo de movilizar la economía. Para ello, es necesario que el Estado pierda su autoridad como decisor económico y se limite a garantizar las condiciones fundamentales para que el mercado funcione. En el camino hacia el establecimiento de este sistema económicopolítico, resulta ineludible la aparición de excesos insoportables que acarrean consecuencias por las que nadie responde. Ante dicho panorama, es inevitable el entrecruzamiento entre lo político y lo disciplinar, es allí donde la práctica que pretende reducir la salud al equilibrio homeostático de la biología, de los procesos cognitivos o de la conducta, deja por fuera lo que el sujeto tiene por producir sobre su sufrimiento.
\end{abstract}

Palabras clave: Poder político, mercado, sistema, vida biológica.

Abstract

In the West during the late eighteenth century the gradual insertion of political power in the the population biological life is experiencing, this control is exercised following an economic aim. Therefore it is necessary for the State to lose its authority as an economic decision maker and is limited to ensure the market performance. On the way to the establishment of this economic and political system, it is inevitable the emergence of intolerable excesses that have consequences for which no one answers. Given this context, it is inevitable the interbreeding between the political and the discipline or practice which aims to reduce health by homeostatic balance of biology, cognitive processes or behavior. This leaves out what the subjects could produce about their suffering.

Keywords: Political power, market, system, biological life.

Résuné

Dans l'Ouest à la fin du XVIIIe siècle, l'insertion progressive du pouvoir politique dans la vie biologique de la population connaît, il est un contrôle qui est exercé dans le but de mobiliser l'économie. Il est donc nécessaire que l'État perde son autorité en tant que décideur économique et est limitée pour assurer les conditions de base pour que le marché fonctionne. Sur le chemin de la mise en place de ce système économique et politique, il est inévitable l'émergence des excès intolérables qui ont des conséquences pour lesquelles personne ne répond. Compte tenu de ce scénario, il est inévitable métissage entre la politique et la discipline, qui est où la pratique qui vise à réduire la santé par l'équilibre homéostatique de la biologie, des processus ou des comportements cognitifs, quitte à ce que le sujet est à produire sur leur souffrance.

Mots-clés: pouvoir politique, système de marché, la vie biologique. 



\section{El otro como objeto: biopolítica, transpolítica y diagnóstico*}

América Celeste Guevara Parra

\section{INTRODUCCIÓN}

El presente artículo es la recapitulación del proyecto de investigación titulado "La biopolítica en la era traspolítica: la violencia del diagnóstico", que fue construido como resultado de las discusiones y trabajo continuo, crítico y riguroso en el interior del semillero "sujeto y psicoanálisis", especialmente dentro de la línea de investigación "El discurso médico y las terapias cognitivo conductuales, la exclusión de la subjetividad en la psicología clínica contemporánea". Se pretende con este enfatizar en cómo se hacen aparecer, a través de prácticas discursivas, los diferentes valores de referencia que son adoptados por la sociedad en un momento particular y según interés específico. Estos valores no responden necesariamente a criterios científicos como comúnmente es presentado, sino a criterios económicos y políticos que favorecen a ciertos sectores.

Ahora bien, es en dicho contexto en el que son situados los fenómenos catalogados como biopolíticos y transpolíticos. En primer lugar, biopolíticos en la medida que involucran prácticas disciplinarias que se autorizan, en nombre de un saber científico, para tomar decisiones sobre la población. Estas decisiones están basadas en el ideal contemporáneo de normalidad, o sea, en la idea de salud con la que el deber no es solamente sobreponerse a la enfermedad, se trata de ir más allá. Desde un enfoque preventivo, se hace aparecer a un nuevo tipo de consumidor que debe esforzarse por adoptar "conductas saludables" con el cometido de potencializar la vitalidad. Este enfoque favorece particularmente a la industria que desarrolla los medios con los cuales supuestamente se consigue estar saludable. Por medio de dicho poder ejercido sobre la población, se interioriza un discurso sobre la salud, la higiene y la vitalidad y en consecuencia, los ciudadanos se convierten en policías de sí mismos.

En segundo lugar, se habla de fenómenos transpolíticos para hacer referencia a que la biopolítica contemporánea (nuevas formas de ejercer control sobre la vida) trasciende los límites de lo político y lo simbólico. En el panorama actual, las instituciones se debilitan, pierden el respeto que tradicionalmente se les tenía como autoridad y ceden su lugar a las fuerzas del mercado. Dichas fuerzas hoy se valen del discurso de la mundialización, la tecnificación y digitalización, como plataformas

El presente texto se enmarca dentro del desarrollo de la Maestría en Antropología Social realizada por la autora. 
desde las que se promete suplir los deseos de los consumidores. No obstante, todo lo que se ofrece es efímero, transitorio y desechable, lo anterior explica el auge del constante desengaño, angustia y decepción que llevan a seguir buscando, por la misma vía, algo que cumpla con las promesas de satisfacción, como esta es una promesa imposible de cumplir, una vez algo deja de funcionar es desechado y el ciclo se repite.

Este sistema que produce necesidades y ofrece al mismo tiempo los medios para suplirlas, es auxiliado por las disciplinas científicas que ponen en funcionamiento diferentes prácticas para hacer aparecer objetos factibles de manipulación y control. En el caso particular de las llamadas "Ciencias de la Salud" se hace uso de diagnósticos inscritos en manuales estadísticos. Ahora bien, con el uso de categorías diagnósticas, se producen sujetos que son tratados como cosas, esto debido a que la enfermedad o anomalía (el diagnóstico) pasa a remplazar al enfermo. Bajo estas consideraciones, la pregunta que resulta orientadora es ¿cómo se justifican ciertas prácticas clínicas como científicas cuando estas obedecen a pedidos que son de otro orden (económico-político, por ejemplo)?

Ahora bien, con el objetivo de responder a dicha cuestión, son considerados especialmente dos enfoques, a saber, la investigación con el psicoanálisis y la investigación arqueológica propuesta por Michel Foucault. En primer lugar, partiendo de la perspectiva psicoanalítica, se quiere enfatizar que este trabajo se sostiene por una epistemología basada en lo singular y no desde disciplinas "basadas en la evidencia" que buscan pruebas científicas y datos objetivos que soporten sus estamentos. Tener en cuenta la singularidad en la investigación implica, por el contrario, considerar aquello que es desechado, por no estar debidamente descrito en un sistema científico particular, como punto de apertura. Lo anterior requiere que la investigación parta desde una posición de no saber, es decir, ignorar lo que se conoce con relación a un tema en particular para que en ese lugar emerja algo inédito.

El segundo aspecto tenido en cuenta junto a la investigación con el psicoanálisis es lo que Foucault denomina carácter histórico-crítico, a priori histórico o arqueología del saber. Esta forma de investigar consiste en interrogar a la historia por sus condiciones de emergencia, es decir, por los juegos de verdad en los que el mismo sujeto se plantea como objeto de un saber posible (en tanto loco, enfermo o delincuente) por medio de prácticas como la psiquiatría, la medicina clínica y la penalidad. Se destaca además desde esta perspectiva, que ser crítico implica renunciar a aceptar como verdadero aquello que una autoridad dice que es verdad solo por el estatus que esta porta. Foucault propone rastrear aquello que hace posible que lo enunciado por dicha autoridad tenga efectos de poder y aquello que permite que prácticas coercitivas sean justificadas por un orden como racionales. Para este cometido resulta indispensable elucidar las múltiples relaciones que es necesario sean efectuadas para que un elemento irrumpa como verdadero en determinada época y en ciertos tipos de discursos. 
Ahora bien, siguiendo estas propuestas como enfoques orientadores, se hace posible la construcción de tres capítulos que representan los temas principales del proyecto de grado y dan cuenta del deseo por saber y profundizar en relación con las formas contemporáneas de biopoder. En el primero, dedicado a la noción foucaulteana de biopolítica, se hace un rastreo que abarca tantas propuestas que, desde los griegos, hacen énfasis en el estatuto simbólico del ser humano, como a distintas maneras de ejercer control biopolítico sobre la población y el sujeto. Con esto se busca enfatizar en ciertas maneras de ejercer el poder sobre la vida dependiendo del discurso predominante en cada época. Se llama la atención especialmente sobre la pérdida del sentido del hombre como aquel capaz de una existencia política, este pasa en su lugar a convertirse en objeto para la política.

En el segundo capítulo es entendida la noción de trasnpolítica como aquello que trasciende los límites que alguna vez funcionaron como principios orientadores para los hombres. El resultado de lo anterior es la emergencia de un mundo que produce irresponsables bajo la insignia de que se es cliente y como tal se tiene derecho a demandar a que otro responda en su lugar. En este caso, es la economía la que responde con artículos que prometen llenar el vacío que hay en cada sujeto. Finalmente, el tercer capítulo está dedicado a prácticas relacionadas con las denominadas ciencias de la salud que, en alianza con la economía de mercado, contribuyen por medio de diagnósticos a producir sujetos-objeto, enfermedades para las cuales hay una receta farmacéutica esperando. Al respecto, se destaca que para "curar" una enfermedad primero hay que producirla. Aquí nuevamente cobra sentido la pregunta de si estas prácticas responden realmente a un saber científico. Finalmente, es mencionada la posibilidad que plantea el psicoanálisis para lidiar con la incertidumbre y el sin sentido en el que se ven envueltos los seres del lenguaje.

\section{BIOPOLÍTICA}

Para introducir el término foucaulteano biopolítica resulta necesario en primer lugar hacer referencia a la época clásica, en este sentido, para los griegos la vida es considerada como sujeto de la política. Luego, este modelo es relegado por otros que proponen distintas formas de lidiar con la cuestión del poder y la vida y terminan por posicionar un prototipo de sociedad en el que, en lugar de ser sujeto político, se es objeto de la política. Teniendo en cuenta lo anterior, se considera indispensable a los propósitos del texto mencionar ideas tanto de Platón como de Aristóteles para quienes la sociedad debe organizarse según un esquema similar al usado para entender la vida biológica: Platón (1872) hace alusión a que la polis es análoga al organismo dado que está compuesta por una pluralidad de partes menores con funciones propias e insustituibles. Por ejemplo, así como no se espera que el corazón supla las funciones del hígado o de ningún otro órgano, tampoco se espera del gobernante nada distinto a que gobierne en virtud de la sabiduría, virtud que no es aludida ni en los guerreros ni en el pueblo, pues de los primeros, se requiere que sean valientes, y de los segundos, que ejerzan la templanza o el control de sus instintos. 
Se destaca también desde los griegos, particularmente desde Aristóteles (2002), al hombre como único animal capaz de existencia política, esto gracias a que se le ha concebido el don de la palabra con el que es posible la construcción de un universo simbólico. Sin embargo, aquel hombre que tiene la capacidad de relacionarse políticamente y gracias a ello de hacerse un lugar, es desplazado de manera constante por distintos mecanismos de poder. Es decir, es minado lo particular que le hace humano, el lenguaje, para enfocarse en la búsqueda de elementos comunes como seres vivientes susceptibles de ser homogenizados.

Foucault a lo largo de su obra hace alusión a ciertos momentos que ilustran ese paso, entre ellos menciona el mercantilismo y el contrato como formas de gobernar que fueron predominantes en su tiempo. Por un lado, el mercantilismo (1981) se constituye como una alternativa ante la dificultad del monarca de mantener el poder haciendo uso de la fuerza, aquel se veía amenazado, tanto desde el exterior por quienes deseaban ocupar su lugar como desde el interior, pues los súbditos no encontraban en la figura del rey mérito alguno para consentir su mandato. Es así como, para mantener el poder, en lugar de valerse de la fuerza y de los castigos, se hace funcionar un conjunto de tácticas (entre ellas la estadística) en el interior mismo de lo que se desea gobernar (la familia, la escuela, el convento, los súbditos).

No obstante, una vez fijada una forma interior de gobernar, es necesario poner límites al poder absoluto del monarca y para ello son fijadas las leyes del derecho. Se hace entonces factible que emerja con Jean-Jacques Rousseau (1988) la teoría del contrato social. Rousseau menciona la importancia de establecer un acuerdo común donde por consenso es escogida una persona en representación de los intereses del pueblo, este último a su vez acuerda unas normas morales y se somete a sus propias leyes. A partir de entonces, el conjunto empieza a ser más importante que sus partes y por lo tanto no será permitido que ninguno de los miembros oponga su voluntad, es decir, será necesario sacrificar la voluntad individual a favor de la masa. En este sentido, es posible argüir que el papel fundamental del derecho es el de fijar la legitimidad del poder que en este caso en lugar de aparecer como dominación arbitraria de un monarca, surge como la obligación legal de la obediencia, es este el efecto del contrato, sostiene la ilusión de libertad como subordinación a la ley en vez de un hombre.

En un gobierno ahora no solo interno sino que además se ejerce en nombre de la voluntad general, es factible el funcionamiento de una mecánica que se ocupa de los cuerpos y de lo que estos producen, a esta mecánica Foucault (2005) la llama poder disciplinario. La disciplina se ejerce por medio de la vigilancia continua y un discurso particular que ya no es el del derecho sino el de la normalización (la disciplina aumenta las fuerzas del cuerpo en términos de utilidad y las disminuye en términos de obediencia) y donde más que obedecer a la ley, se está a merced del sistema económico. Dicho poder normalizador funciona estableciendo un circuito entre el saber que se le supone a la ciencia y el poder que adquieren las disciplinas (que buscan ser reconocidas como científicas) por el prestigio otorgado gracias a ese saber 
que se les supone. Las disciplinas, se encargarán de poner en funcionamiento una vigilancia continua en colegios, hospitales, fábricas, cárceles y demás instituciones y de utilizar técnicas que permiten inducir efectos de poder sobre los vigilados. Aquel ejercicio del poder sobre el cuerpo, permite la introducción controlada del mismo en los aparatos productivos, fenómeno indispensable para que el capitalismo tenga lugar (Foucault, 2010).

Junto a la disciplina que se ocupa de los cuerpos, se pone en funcionamiento otra tecnología de gobierno, esta vez denominada por Foucault (1998) como biopolítica. Ahora bien, Foucault usa la palabra "biopolítica" para señalar aquello que permite convertir fenómenos relativos a la vida biológica en dominio del cálculo y transforma al poder-saber, es decir, al poder que es otorgado a la ciencia debido al prestigio derivado del saber que se le supone, en agente de cambio para esa vida. Con esta instancia regularizadora el objetivo ya no son los individuos sino la población, aquella se hace aparecer haciendo uso de tasas de natalidad, mortalidad, fecundidad, longevidad y movilidad que, al relacionarlas con mecanismos de costos y producción, están ligadas a la consolidación del liberalismo a partir del cual irrumpe la economíapolítica como razón gubernamental moderna (Foucault, 2010). Entonces, para abordar los fenómenos de individualización, por un lado, y de población por otro, se acude a toda una tecnología disciplinaria del cuerpo y a una tecnología regularizadora de la vida, juntas contribuyen a transformar la vida en objeto utilizable para el poder.

Las autoridades políticas en alianza con otras prácticas asumen la tarea de administrar la vida en nombre del bienestar de todos (precepto que es posible gracias al contrato social) y para ello se apoyan en técnicas adoptadas por la psicología. En este sentido, Foucault (2010) menciona particularmente la propuesta de Skinner que contribuye a que el sujeto de la economía, el Homo œconomicus, aparezca como un elemento manejable (gobernable) que responde sistemáticamente a las modificaciones introducidas en el medio. Esto hace posible que, con el behaviourism, se adopten técnicas o instrumentos que permiten ejercer poder sobre el cuerpo. Por consiguiente, aunque estas técnicas se justifiquen como terapéuticas, su objeto último es contribuir al mantenimiento de un orden para el que el sujeto no es más que un objeto factible de manipular y el psicólogo no es más que un funcionario.

Una de las industrias que resulta favorecida con el panorama mencionado es aquella que gira alrededor del negocio en que se ha convertido la salud. El foco en terminos de políticas públicas se ha desplazado de la administración de la enfermedad, hacia la optimización del cuerpo sano, por ende, las autoridades buscan inculcar en la población la adopción de una "ciudadanía activa" que integre la salud como valor fundamental. Según el sociólogo británico Nikolas Rose (2012), el efecto buscado es el de producir consumidores que exijan y usen activamente la medicina. Muy comprometida con esta labor se encuentra la Psicología de la Salud que concibe a la salud como el producto de procesos biológicos, ambientales y comportamentales e incorpora la metodología, los principios y los conocimientos de la actual Psicología Científica para desarrollar métodos que permitan el mantenimiento de estilos de vida saludables (American Psychological Association, 2015). 
En el camino hacia la potencialización de la salud, los científicos se valen de dispositivos técnicos y tecnológicos que permiten introducir la vida en el nivel molecular, por ejemplo, con el perfeccionamiento de la imagenología es posible traspasar los tejidos como nunca antes. El resultado es que el médico ya no necesita de su intuición para saber sobre la enfermedad, su mirada ha sido remplazada por la Resonancia magnética y la Tomografía axial computarizada (lo que hace pensar si con el tiempo, argumentando la importancia de eliminar el error humano, las maquinas permitirán remplazarlo por completo). Ahora bien, dado esta escalada de avances técnicos y tecnológicos, la mente es localizada en el cerebro, en esta vía, como consecuencia de equiparar lo psíquico a lo orgánico, emerge un campo factible de intervención a través de psicofármacos. En otras palabras, surge un terreno ideal para la explotación bioeconómica, de ahí que para Rose (2012) la neuropolítica sea la nueva forma de biopolitica.

Por consiguiente, y a modo de conclusión, es posible aludir que el biopoder es entendido como efecto de la alianza que se establece entre economía y ciencia, esta alianza hace factible que el poder sobre la vida, sobre los cuerpos y sobre la población, no sea exclusivo de las instituciones vinculadas al Estado, participan otras, como la psicología o la medicina, que contribuyen con un conjunto de dispositivos y técnicas de dominación. El resultado final es la reducción del sujeto del lenguaje (que gracias a ello es capaz de existencia política y por lo mismo de diferenciarse de los animales) a un objeto factible de ser disciplinado a favor de un orden que es principalmente económico.

\section{TRANSPOLÍTICA}

Otro tema clave a los fines de la investigación es la noción de transpolítica desarrollada por el filósofo y sociólogo francés Jean Baudrillard. Con el propósito de introducir el termino, es necesario retomar lo mencionado anteriormente en relación con la pérdida gradual del poder, en primer lugar, la pérdida del poder absoluto que recaía sobre el cuerpo del monarca y, en segunda instancia, del poder estatal en la medida que lo cede a la economía. En este sentido, se pretende destacar como fenómeno transpolítico, el declive en los límites o principios estructuradores antes simbolizados por dichas figuras de autoridad (el rey y el Estado).

Ahora bien, el dilema se encuentra más allá del hecho que las instituciones estatales ya no tengan la exclusividad del poder que alguna vez tuvieron, hoy no solo ceden el poder a las fuerzas anónimas de la economía, sino que a la vez y como resultado de lo anterior, pierden respeto y credibilidad. Es decir, como en las formas posmodernas de ejercer el poder, la preocupación no es mantener un marco institucional fuerte, estable y duradero, o en otras palabras, como el Estado se declara impotente para responder, las nuevas instituciones nacen con el estigma de la transitoriedad, en consecuencia, aquellas ya no sirven a los hombres de marco de referencia sobre el cual estructurar sus proyectos de vida. 
Para el sociólogo polaco Zygmunt Bauman, interesado en el análisis de los fenómenos que tienen lugar en lo que el llama modernidad líquida (palabra usaba para referir la falta de solidez de los valores contemporáneos), dicho desmantelamiento de controles políticos en lugar de hacer libres a los seres humanos, los esclaviza a las fuerzas irresistibles del mercado (Bauman \& Tester, 2002). Por su parte, el sociólogo Gilles Lipovetsky (2011), menciona que el contexto actual, caracterizado por mundialización y la ideología de la salud, le da cabida a un régimen de "autoservicio" donde la "plenitud personal" es lo que se impone como ideal último. En un contexto tal, el hombre busca medidas inmediatas para problemas inmediatos y no una vuelta al orden o a referentes estructuradores.

Lipovetsky (2011) agrega que el sistema de derechos humanos hace imposible estar a la altura de ideales como libertad, igualdad y felicidad para todos. Se trata entonces de un conjunto de promesas que resultan imposibles para quienes gobiernan y en consecuencia la acción política parece muy calculada, injusta y siempre por debajo de lo que idealmente se espera y exige. Como resultado de lo anterior, para Bauman (2001), los ciudadanos se sienten poco afectados por la vida política, los hombres se alejan de los asuntos tradicionalmente considerados como públicos y ese lugar es ocupado por lo privado. En lo público, ya no se buscan causas comunes sino la posibilidad de estar "interconectado" y para ello se comparte la intimidad con el mundo. Entonces, de acuerdo con el nuevo contexto mundial, caracterizado por la liberación y el predominio del sistema financiero en la economía que reduce de manera drástica los márgenes de maniobra del poder público, se produce un amplio desinterés y desilusión por la política a la vez que incrementa la búsqueda de "alegrías privadas" apoyada en el estímulo sistemático de las novedades comerciales, el ocio y la plenitud individual.

Ahora bien, en paralelo con el advenimiento de una sociedad que estimula el crecimiento de su "yo narcisista", entran en juego cambios en diferentes sectores que reducen al mínimo el sentido que solían tener los eventos y las cosas. Un ejemplo es el mismo sentido del amor, este es despojado de toda imaginación metafórica dado que el narcisismo desvincula al amor físico de cualquier tipo de compromiso tanto social como personal (Sennett, 2011), por eso, la pornografía no puede ofrecer más que significados superfluos (Baudrillard, 2000).

Otro ejemplo citado en este caso por Baudrillard (2000) tiene que ver con poner en suspensión la misma idea de hombre. El sentido de ser humano está en peligro si se tiene en cuenta que, en la continua búsqueda de un ideal genético, emergen "recetas" que tienen el cometido de deshacerse de aquello que resulte un obstáculo a ese ideal, o sea, todo lo que sea "demasiado humano" (deseos, defectos, neurosis, locuras, sexualidad...), en consecuencia, "los seres singulares pasan a ser copias idénticas de los otros" (p. 21).

Baudrillard (2000) también se refiere a la realidad virtual como aquella que mina el sentido. El mundo de las redes y la información emite los acontecimientos en tiempo real, todo circula de forma infinita e inmediata haciendo que las cosas tengan 
todos los significados posibles, al final, el resultado es la misma ausencia de sentido. Lo anterior es muestra de cómo en un mundo que pretenden alcanzar la perfección en todas las áreas, que pretende almacenar y memorizar todo, no hay finalidad diferente a la eterna reproducción de lo mismo, es decir, a la continua reproducción del sistema actual.

En suma, cuando los límites son indistinguibles se pierde el sentido mismo de las cosas, el sentido de lo público y lo privado, el sentido del sexo y del amor, el mismo sentido de lo humano y de los acontecimientos. Esto pasa en una sociedad que busca la perfección y que en ese camino deja por fuera aquello que hace que las cosas y los sucesos sean únicos, desdibuja las coordenadas que fijaban su sentido. Es así como los ideales que antaño encausaban la vida de los hombres tienden a desaparecer, la preocupación hoy es ajustarse a las exigencias contemporáneas de alcanzar la plenitud personal. A este campo de variaciones sin márgenes que mina el sentido, es a lo que Baudrillard da el nombre de transpolítica.

\section{EL DIAGNÓSTICO Y LA VIOLENCIA DEL LENGUAJE}

Ahora bien, luego de mencionar en primera instancia al interés de la ciencia por hacer emerger la vida como un objeto factible de manipular y predecir permitiendo ajustarlo a lo exigido por los sistemas que son sobre todo económicos, $\mathrm{y}$ de hacer énfasis en el segundo apartado en cómo, en la búsqueda constante por la perfección en distintos terrenos, aquello que identifica a la vida humana misma se vacía de sentido, a continuación se hace referencia a prácticas clínicas guiadas por el diagnóstico como aquellas que contribuyen al mantenimiento de este sistema al que solo le interesa su propia reproducción.

Las clasificaciones de trastornos mentales proporcionan descripciones con las cuales se compara a un sujeto, lo anterior con el fin de establecer si pertenece a una categoría en particular y actuar en consecuencia. Entonces el diagnóstico (en tanto intento de atrapar el psiquismo) precede a la decisión del especialista de usar determinada terapia (usualmente enmarcada en los principios de las terapias cognitivo - conductuales) o en la utilización de fármacos con los que se reduce lo psíquico a un proceso biológico.

Estos diagnósticos de enfermedad mental se soportan en manuales clasificatorios que en un primer momento acudían a corrientes teóricas (particularmente al psicoanálisis) para plantear sus presunciones, pero actualmente su base es la estadística y el consenso. Por ejemplo, en relación con el Manual diagnóstico y estadístico de los trastornos mentales (DSM) es aludido que: "el DSM...se pretende "a-teórico" y su redacción se mide con la escala de la norma estadística y de la opinión pública” (Caroz, 2011, p. 10).

Al respecto, es necesario decir en primer lugar que resulta contradictorio basar la estadística como criterio de lo objetivo, pues los datos que con ella se obtienen no son hechos sino construcciones sociales donde alguien decide qué contabilizar, en esta 
vía, como la información que se "obtiene" se puede usar a conveniencia, termina por convertirse en un instrumento ideal para la manipulación. Es entonces una elección que es más política (ideológica) que científica (González \& Pérez, 2007). En segundo lugar, resulta curioso que a pesar del auge de campañas de promoción de la salud y prevención de la enfermedad, en cada versión del DSM el número de entidades clasificatorias en lugar de disminuir aumente: "en el primer DSM publicado en 1952 se establecen 106 categorías diagnósticas, en el DSM-II (1968) pasan a ser 182, en el DSM-III (1980) a 265 y en el DSM-IV (1994) a 297” (Pundik, 2010, p. 25), con la llegada del DSM-5 se traspasa la barrera de los 300 (Begley, 2013).

Dada esta escalada de categorías, ya no es fácil percibir si son antes los trastornos mentales (los problemas) y después los psicofármacos (las soluciones), como cabría suponerse, o si son antes los psicofármacos y después los trastornos. Lo anterior no significa que sea la existencia de los psicofármacos la causa de que se tenga problemas, pero sí que los problemas que tiene la gente son inscritos en la forma de trastornos mentales de supuesta base biológica remediable precisamente con fármacos (González \& Pérez, 2007). En este contexto, se sitúa el caso de la depresión que curiosamente, antes considerada como un diagnóstico raro, desde los años noventa se vuelve "una epidemia" resultado del auge del prozac, "este boom de la depresión empezaría con el DSM III y los nuevos antidepresivos” (p. 62).

Ahora bien, se hace énfasis de forma crítica en esta situación no porque se esté en contra del uso de medicamentos, sino con el objetivo de llamar la atención sobre la importancia de tener en cuenta que una reducción de lo psíquico a lo orgánico disuelve lo singular, lo propio de cada sujeto, en universales clasificatorios o químicobiológicos que se desentienden del caso por caso.

En relación con lo anterior, para el psiquiatra y psicoanalista francés Jean Clavreul (1983), el discurso médico interpreta el sufrimiento mismo como un signo entre otros, el médico rechaza lo que pueda enunciarse como consideración sobre el mal, la culpabilidad, la vergüenza y el pudor y es excluido que el enfermo oponga sus razones a la razón médica. No obstante, la razón que se impone en su lugar, o sea la razón del médico, está menos del lado del enfermo que de responder ante sus pares, es decir, ante la jurisdicción de la "Orden de los médicos", se apaga en consecuencia la voz del sujeto para imponer la del otro "dueño de la verdad".

Por lo anterior, se concluye que el diagnóstico es violento principalmente por poseer un estatuto de poder que no merece si se tiene en cuenta su calidad intrínseca, es decir, si se tiene en cuenta que obedece a criterios basados en ideales sociales de adaptación que las distintas disciplinas han ayudado a construir y posicionar; que apela a descripciones sin teoría que juzgan comportamientos en lugar de recurrir a la construcción de una episteme para intentar explicar el sentido de esos comportamientos. Otra razón, que será ampliada en el siguiente apartado, tiene que ver con el proceso mismo de otorgar palabras a las cosas, por ahora se alude que otorgar una palabra (como un diagnóstico por ejemplo) a algo o a alguien, es un ejercicio de violencia en la medida que se hace decir lo que aquello por sí mismo no dice. 


\section{¿COSAS” O CONSTRUCCIONES DISCURSIVAS?}

Desde la perspectiva que se propone en esta investigación, los trastornos mentales son entendidos no como entidades de base biológica (a priori al sujeto), como gran parte de la actual clínica pretende hacer ver, sino son considerados construcciones que emergen en la intercepción entre un discurso y un cúmulo de relaciones que son tanto políticas como sociales y económicas. No obstante, que no se entienda como este resultado de un desequilibrio neuroquímico no quiere decir que no sea real, que no tenga lugar, sino que obedece a una realidad distinta, la realidad que le otorga el discurso en que se inscribe y no aquella que le otorga el cerebro. En ese sentido, resulta indispensable sentar una posición que sin oponerse rotundamente al uso del fármaco (no se niega su utilidad en algunos casos), si defiende la necesidad de optar por una clínica en la que ni el fármaco ni la etiqueta diagnóstica reemplacen la palabra.

Para tal cometido, en primera instancia se recurre a Foucault para quien, en $\mathrm{La}$ arqueología del saber (2007), las cosas son previas al discurso, jamás lo preceden. Foucault designa como objeto al producto de atribuir una palabra a la cosa, es decir, los objetos son formaciones discursivas. Ahora bien, para que surja un objeto es necesario que se pongan en juego un haz de relaciones (institucionales, relativas a procesos económicos y sociales, a formas de comportamiento, a sistemas de normas, a tipos de clasificación...). Pero este haz de relaciones, como lo llama el autor, no es lo que define la constitución interna del objeto sino lo que le permite aparecer.

Lo anterior supone que se le da sentido a la realidad según la naturaleza de un sistema estructurado como lenguaje, en otras palabras, se pone en juego un andamio conceptual que vincula las palabras (es decir, ficciones) con las cosas. En Las palabras y las cosas" Foucault (1968) lo resume de la siguiente manera: "el nombre propio no es más que un artificio" (p. 19), artificio que permite cerrar el espacio del que se habla y el que se contempla (o sea palabra y cosa) uno en otro con toda comodidad "como si fueran mutuamente adecuados" (p. 19.). Por tanto, los objetos que emergen en el discurso a pesar de tener como característica la dispersión (surgen como producto de un entramado de relaciones), son velados por una superficie que los hace ver como si fueran una unidad (nuevamente, como si palabra y cosa fueran lo mismo). Entonces, para Foucault el discurso es más que un conjunto de signos, es una práctica con reglas propias que lo hacen excluyente. Cuando se entiende esto se disipa el lazo que une las palabras con las cosas y aparece en su lugar el objeto no como unidad sino como efecto de dispersión. Ahora bien, como enfatiza Foucault (1968), los discursos "verdaderos" no reconocen la voluntad de verdad que los atraviesa y por ende olvidan que los objetos que forman no son las cosas.

En el mismo sentido es posible mencionar lo dicho por el filósofo alemán Friedrich Nietzsche (1996) en Sobre verdad y mentira en sentido extramoral. En el texto el autor enfatiza sobre cómo el hombre ha construido todo un sistema de abstracciones que supone ciertas, dice que incluso ha definido niveles diferentes 
para determinar lo verdadero: discrimina por ejemplo el conocimiento científico valorándolo sobre otros tipos de conocimientos (como el conocimiento "común" por ejemplo). El filósofo argumenta lo anterior refiriendo que los hombres crean conceptos para tratar de entender los objetos circundantes, no obstante, cuando se olvida la naturaleza lingüística de este proceso de categorización, se desconoce estar en contacto con invenciones en lugar de la "esencia" misma de las cosas. Por lo tanto, así como Foucault, también Nietzsche concluye que arbitrariamente se termina equiparando la palabra a la cosa. En esta vía, se alude finalmente que la "verdad" no proviene de lo que son las cosas en sí, "las verdades son ilusiones de las que se ha olvidado que lo son" (Nietzsche, 1996, p. 25).

Cabe anotar que el hombre mismo es el punto de referencia desde el cual los seres humanos nombran las cosas, es decir, las categorizaciones que hace de los objetos son antropomorfas. Pero teniendo en cuenta que los sentidos y la manera como estos captan el mundo varían significativamente de una especie a otra, no es solo injusto sino absurdo asumir que la percepción humana es la correcta, de manera que si no hay percepciones correctas tampoco existen verdades absolutas. Además, al dejar de lado que las percepciones también varían entre individuos, se saca al sujeto de la escena científica para llegar a un conocimiento "objetivo" que, como plantea Nietzsche (1996), no es posible.

Estas asunciones son claves en la medida que permiten pensar desde una perspectiva crítica las prácticas que tienen lugar sobre los sujetos y sobre grupos sociales particulares. Así por ejemplo, a pesar de que la psicología y la psiquiatría excusen su práctica refiriendo que las enfermedades mentales son descubiertas gracias al arduo trabajo de la investigación científica, lo que es puesto en relieve, haciendo una analogía desde las ideas de Nietzsche y de Foucault, es que los trastornos mentales, lejos de ser entidades de base biológica, son objetos construidos dentro de un marco discursivo que tiene sus propias reglas de inclusión y exclusión. Algo similar podría decirse respecto a las ciencias sociales, especialmente la antropología que en siglo pasado toma conceptos derivados de los principios de la ley de la evolución y de la selección natural para entender la situación de naciones que, según esta mirada, no habían alcanzado estadios superiores de desarrollo, por ejemplo las sociedades latinoamericanas fueron catalogadas como "sociedades infantes" y por ello susceptible de ser intervenidas o "ayudadas" por sociedades mayores, idea que legitima el intervencionismo de las potencias (Runge \& Muñoz, 2005).

Es decir, las disciplinas no son ajenas a un marco discursivo que define, entre otras cosas, quién puede y en dónde hablar, define también el lenguaje técnico para utilizar al referirse a los fenómenos e incluso cuál es la realidad que sirve como punto de comparación para decidir, por ejemplo, quién es "normal" y quién no, también define los procedimientos que supuestamente ayudarán a eliminar el síntoma o la enfermedad misma, o los signos de degeneración de una "raza", tiene en últimas el poder de decidir cuál es el "discurso verdadero" en relación con un fenómeno particular. En fin, con lo anterior se pretende resaltar que las entidades son discursos 
(representaciones que surgen bajo un procedimiento discursivo) susceptibles de ser analizadas como tales.

\section{LENGUAJE COMO FUNCIÓN}

Ahora bien, teniendo en cuenta la importancia de abrirle un espacio al análisis de los fenómenos, en tanto, construcciones discursivas, resulta de particular interés referirse a la propuesta de Sigmund Freud dada la relevancia que se le da desde el campo del psicoanálisis al lenguaje. Particularmente, se hace referencia a la lectura que hace, por medio del texto "Función y campo de la palabra y del lenguaje en psicoanálisis" (2009), uno de los autores más influyentes en el campo, el psiquiatra y psicoanalista francés Jacques Lacan.

En la obra en mención, es referido que la genialidad de Freud recae en que concibe la vida anímica del sujeto estructurada como un lenguaje. Por consiguiente, la tarea del analista es la de entender a qué parte del mismo está confinado el término significativo para contribuir con una puntuación afortunada que ayude a dar sentido a ese discurso. Por otro lado, Lacan rescata la exigencia de Freud de acudir a la memoria, sin embargo, aclara que no se trata de una memoria biológica sino histórica. Es decir, se acude a la historia del propio sujeto, historia que está a la espera de ser escuchada por otro, "es ciertamente esta asunción por el sujeto de su historia en cuanto que está constituida por la palabra dirigida al otro la que forma el fondo del nuevo método al que Freud da el nombre de Psicoanálisis" (2009, p. 249).

En este sentido, a diferencia de una etiqueta que alguien otorga desde una posición de saber, avalado por una institución y en función del discurso de "lo verdadero", el arte del analista consiste, no en adscribir un veredicto sino todo lo contrario, en suspender las certidumbres del sujeto "hasta que se consuman sus últimos espejismos" (Lacan, 2009, p. 244). Esto implica tomar un camino distinto al que se ha recorrido siguiendo el discurso de la normalización y el diagnóstico, por el contrario, desde la clínica psicoanalítica se busca que el sujeto pueda de alguna manera simbolizar aquello no-dicho y esto supone poner en cuestión lo que otros afirman sobre él.

Ahora bien, Freud es conocido por conceptualizar el inconsciente como aquello que estructura y dirige la vida psíquica de los hombres. Por lo tanto, se enseña al sujeto a reconocer como su inconsciente su historia, esta entendida como hechos inscritos en cierto sentido o censurados en cierto orden. No obstante, aquello que se olvida, aquello no-dicho, resalta Lacan (2009), se recuerda en actos, en sueños, en el chiste, pero también emerge en forma de síntoma. Este último surge ante la ausencia de la palabra, se dice que aquel es resuelto recurriendo a un análisis del lenguaje, pues "el mismo está estructurado como lenguaje" (p. 260).

En relación con este último, el psicoanálisis ha tenido que lidiar con tres paradojas. En primer lugar, con la locura, la palabra renuncia a hacerse reconocer, ante esto emergen los estereotipos de un discurso que habla en nombre del loco. En segundo 
lugar, con las neurosis, aquí la palabra es expulsada del discurso concreto que ordena la conciencia, entonces (como se mencionó) se habla en el síntoma. Finalmente, con el sujeto de la civilización científica, sujeto que pierde su sentido en las objetivaciones del discurso que lo enajena, es esta enajenación la que "encontramos en primer lugar cuando el sujeto empieza a hablarnos de él” (Lacan, 2009, pp. 271-272).

Para el autor en cuestión, el yo de aquel hombre moderno no reconoce la razón misma de su ser en el desorden que denuncia del mundo, la ciencia se le ofrece como salida para olvidar su subjetividad en la medida en que colabora eficazmente en su trabajo cotidiano y lo llena de ocios dándole así la ocasión de olvidar su existencia y su muerte (Lacan, 2009). Por consiguiente, el discurso del hombre "normal" (discurso que promulga la ciencia como deseable) refuerza el muro del lenguaje, dice Lacan, que se opone a la palabra del sujeto de manera similar a la enajenación de la locura donde más que hablar se es hablado.

Para liberar la palabra del sujeto es necesario, desde esta perspectiva, introducirlo en el lenguaje de su deseo (lenguaje primero en el que más allá de lo que él dice ya habla sin saberlo) y en los símbolos de su síntoma, en otras palabras, saber por quién y para quién plantea su pregunta. Por eso, el psicoanálisis solo da fundamentos científicos a su teoría y técnicas formalizando adecuadamente las dimensiones esenciales de su experiencia que son la lógica intersubjetiva y la temporalidad del sujeto, para ello se vale del lenguaje en tanto que su función no es informar (como sí lo es para el discurso médico-científico) sino evocar.

Se quiere puntualizar en la función del lenguaje porque, teniendo en cuenta la perspectiva psicoanalítica aquí mencionada, las palabras resultan indispensables para bordear el síntoma, no con el cometido de eliminarlo sino para, resignificándolo, hacerlo soportable. Esto es importante puesto que la posición asumida está del lado de entender que lo sintomático tiene una función, es una defensa que hace soportable lo insoportable. Freud (1992) en este sentido refiere que "lo ominoso" se da cuando "todo lo que ha estado destinado a permanecer en secreto, en lo oculto, ha salido a la luz" (p. 224).

El síntoma hace parte de lo que constituye ser un sujeto en la medida que emerge en forma de defensa contra eso que busca salir a luz y que es ominoso (insoportable). Por lo tanto, se aboga en este sentido por una clínica que en lugar de ser el medio a través del cual se prescinde del síntoma, propicie en su lugar la construcción de un saber sobre el mismo que cuente siempre con la palabra, posición y deseo del sujeto. Es de vital importancia repensar no solo la clínica sino también las distintas prácticas que desde las ciencias sociales trabajan con grupos humanos y esto supone abrir un espacio para el diálogo en relación con la situación del ser humano en este mundo que hoy no hace más que desplazarlo. 


\section{DISCUSIÓN}

Por medio del texto, el cometido es esbozar el resultado del arduo trabajo llevado a cabo con el fin de integrar, de forma crítica y analítica, fenómenos contemporáneos que afectan la situación en la que es puesto y con la que debe lidiar el sujeto posmoderno. En primer lugar, se hace énfasis en el poder como aquello que va más allá de lo trasmitido desde las instituciones estatales al conjunto de la población, se enfatiza en que la norma de su funcionamiento está implícita en el interior y cotidianidad del cuerpo social y es dictada por sistemas disciplinarios. Por medio de dichos sistemas hoy se expande el blanco de intervención de fenómenos relativos a la enfermedad a un enfoque centrado en la salud. Los profesionales desde distintos campos asumen su rol como expertos en estilo de vida y hacen parte de un campo interdisciplinar que entiende la calidad del bienestar fisico y psicológico como producto de la interacción del organismo con el medio, de ahí que principalmente se abogue por la adopcion de conductas (habitos) saludables.

Desde dicha perspectiva, es definido lo que es deseable esperar en relación con la salud y la enfermedad, es decir, se opera en una lógica (¿moralista?) que supone hacer juicios respecto a cuáles son las formas de vida aceptables y se publicitan intervenciones que permitirían alcanzar esas formas de vida. Lo anterior permite concluir que, en la biopolítica contemporánea, la autoridad en el gobierno del yo somático no son los políticos, son los clínicos cuyo conocimiento especializado supera tanto el diagnóstico como el tratamiento de las enfermedades.

Entonces, el papel particular de distintas prácticas individuales y sociales consiste en velar porque el sujeto (visto como capital) esté en condiciones óptimas para seguir siendo útil a un sistema, para tal cometido se valen de tácticas como el apelar a procedimientos sustentados en diagnósticos. Ahora bien, partiendo de suponer que el diagnóstico de enfermedad mental es un discurso, una manera de representar un fenómeno (entre toda una serie de posibilidades) y no una entidad, la pregunta en la que se insiste es por qué esa manera y no otra. Principalmente se puede argüir que es inconcebible reconocer a la experiencia empírica una condición discursiva, pues esto implicaría darle lugar a otras versiones y, en este sentido, un orden se sostiene solo si consigue instaurar un aparato de exclusión e inclusión de lo que puede ser aceptado como verdadero, en consecuencia, utiliza el mismo discurso para negar la condición discursiva de sus prácticas.

Es así como la construcción de los criterios del DSM se debe más bien a que se obedece a la ideología de la ciencia para escudar ciertas prácticas en curso (económicamente muy favorables para ciertos sectores) que a un triunfo del rigor científico sobre la ideología. Es decir, se fabrican trastornos con base en la ideología de la ciencia con la que se pretende otorgarle a la psiquiatría criterios que le permitan abordar lo mental de forma análoga a como la medicina inscribe lo orgánico. Dado lo anterior, en la medida que la psiquiatría y la psicología participan de forma activa en la construcción y mantenimiento de criterios diagnósticos basados en lo que es 
favorable para el mercado al que están inscritas, se podría decir que aquellas son las creadoras de su propia objetividad.

Por lo tanto, es puesta en duda la idea de diagnóstico en tanto ente al margen del discurso que lo hace existir. El trastorno, en lugar de una entidad natural, es aquí entendido como una construcción discursiva cuya forma es la que los clínicos necesitan para poder tratar los fenómenos mórbidos de la manera que lo hacen. Esto quiere decir que la supuesta objetividad de un diagnóstico descansa en demandas políticas y comerciales dirigidas a disciplinas como la medicina y la psicología. Lo anterior explica el porqué, a pesar de vivir en "la era del bienestar", se multiplican las conductas diagnosticables como patológicas, este incremento beneficia a la industria en que están inscritas gran parte de las terapias actuales.

Mientras los fenómenos relativos a la enfermedad mental sean concebidos en la lógica de la entidad (o diagnóstico) por un discurso que se autoriza como "verdadero" y en el cual quien habla es el clínico (pero no en nombre propio sino en tanto representante de un orden), esta aparecerá como determinada. Es decir, al olvidar que los nombres utilizados para referirse a los fenómenos no son los fenómenos mismos, se cae en el juego de imponer categorías a las cosas con la pretensión de que estas sean absolutas. Por lo tanto, instalar en una persona determinada etiqueta, que pasa a hacer aquello que lo representa, impide que este sujeto pueda hacerse a un nombre propio, que pueda otorgar según su discurso un sentido a su queja, a su síntoma.

Este último se reúsa a ser inscrito en las lógicas de lo absoluto, obedece a lo que es singular al sujeto, a lo in-inscribible en el para todos aplicable a la clínica guiada por el diagnóstico. En esta vía, con el psicoanálisis -a diferencia de la clínica psicológica actual que renuncia a la epistemología para sostener su práctica con estadísticas (eliminando el debate) - se busca la producción de un saber sobre el síntoma, es decir, supone una construcción epistemológica basada en la propia historia del sujeto.

\section{REFERENCIAS}

American Psycholocal Association. (2015, abril 16). What is Health Psychologist? Obtenido de health-psych.org: http://www.health-psych.org/AboutWhatWeDo.cfm

Aristóteles. (2002). La Política. Bogotá: Panamericana.

Baudrillard, J. (2000). Las estrategias fatales. Barcelona: Anagrama.

Bauman, Z. (2001). La sociedad individualizada. Madrid: Cátedra.

Bauman, Z., \& Tester, K. (2002). La ambivalencia de la modernidad y otras conversaciones. Barcelona: Paidós.

Begley, S. (2013, mayo 16). DSM-5: Psychiatrists' 'Bible' Finally Unveiled. Obtenido de Huffpost Healthy Living: http://www.huffingtonpost.com/2013/05/17/dsm-5unveiled-changes-disorders-_n_3290212.html

Caroz, G. (2011). ¿La salud mental existe? Revista Letras, 2, 10-11. Obtenido de letraslacanianas.com: http://issuu.com/uzapuca/docs/letras_2_digital_ocr 
Clavreul, J. (1983). El orden médico. Barcelona: Argot.

Foucault, M. (1968). Las palabras y las cosas. Buenos Aires: Siglo XXI Editores.

Foucault, M. (1981). La gubernamentalidad. En R. F. Castel. Espacios de poder, (pp. 9-26). Madrid: La Piqueta.

Foucault, M. (1998). Historia de la sexualidad (t. 1). México, D.F.: Siglo Veintiuno.

Foucault, M. (2005). Vigilar y castigar. Buenos Aires: Siglo XXI Editores.

Foucault, M. (2007). La arqueología del saber. México, D.F.: Siglo XXI Editores.

Foucault, M. (2010). Nacimiento de la biopolítica. Buenos Aires: Fondo de Cultura.

Freud, S. (1992). Lo ominoso. En S. Freud. Obras completas vol. XVII (pp. 215-251). Buenos Aires: Amarrortu Editores.

González, H., \& Pérez, M. (2007). La invención de los trastornos mentales. Madrid.

Lacan, J. (2009). Función y campo de la palabra y del lenguaje en psicoanálisis. En J. Lacan. (s.f.). Escritos I (pp. 231-310). México, D.F.: Siglo XXI Editores.

Lipovetsky, G. (2011). La sociedad de la decepción. Entrevista con Bertrand Richard. Nietzsche, F. (1996). Sobre verdad y mentira en sentido extramoral. Madrid: Tecnos S.A.

Platón. (1872). El político. En Platón. Obras completas (t. VI, p. 110). Madrid: Medina y Navarro Editores.

Pundik, J.(2010). ElDSM: la Biblia del totalitarismo. Topia, núm. 62, 25-27. Obtenido de topia.com.ar: https://www.topia.com.ar/file/349/download?token=KBBxIU62

Rose, N. (2012). Políticas de la vida. BioMedicina, poder y subjetividad en el siglo $X X I$.

Rousseau, J. (1988). El contrato social. Barcelona: Tecnos S.A.

Runge, A., Muñoz, D. (2005). El evolucionismo social y la educación en Colombia, primera mitad del siglo XX: el cuerpo en las estrategias eugenésicas de la línea dura y de la línea blanda. Revista Iberoamericana de Educación, No 39, 127 168. Medellín, Universidad de Antioquia.

Sennett, R. (2011). El declive del hombre público. Barcelona: Anagrama S.A. 